\title{
Two modified natural in vitro fertilisation (IVF) protocols compared to conventional IVF treatment: Retrospective data from one Danish Fertility Centre
}

\begin{abstract}
Aim of study: Over the last decade, laboratory procedures in in vitro fertilisation (IVF) have improved. Hyperstimulated ovaries cause an overload of surplus embryos. The present study was designed to evaluate the efficiency of two different modified IVF cycle protocols trying to reduce the load of medication used for IVF: simple IVF (S-IVF), Tamoxifen 40 $\mathrm{mg}$ daily from day 2 in the cycle to ovulation induction) and mild IVF (M-IVF), Tamoxifen $40 \mathrm{mg}$ daily and every secondary $150 \mathrm{IU}$ Gonal $\mathrm{F}$ until ovulation induction. The study aims to evaluate their efficiency compared with our conventional IVF (C-IVF) using a short antagonist protocol.
\end{abstract}

Methods: A retrospective cohort study including all patients admitted to IVF for unexplained infertility, tubal factor, and male factor. In all stimulated cycles patients aimed at having fresh embryos transferred and surplus good embryos cryopreserved. All patients were recruited in the same period and allocated to the different treatments on their own request. The study was conducted between June 2019 and February 2021.

Results: In total the study included 976 IVF cycles. 651 cycles from C-IVF, 145 cycles from S-IVF and 180 cycles from M-IVF. Mean age in the groups were comparable. Mean number of eggs retrieved was 6.1 (C-IVF), 1.2 (S-IVF) and 3.0 (M-IVF). Pregnancy rate per fresh transfer was found to be $29 \%$ for C-IVF, $26 \%$ in the S-IVF group and for the M-IVF $20 \%$.

Conclusion: Modified IVF stimulation protocols may be an important step towards a simpler assisted reproductive technology (ART) approach. More tolerable for women, easier and cheaper for patients and society they maintain acceptable clinical pregnancy rates. Large prospective studies need to be performed in the future.

Keywords: Ovarian stimulation, IVF, ART, low dose stimulation, mild stimulation, minimal stimulation, natural IVF, modified natural cycle IVF
Volume 12 Issue 6 - 202I

\author{
G] Almind,' A Abraham-Zadeh,' E Faerch,' F \\ Lindenberg,' S Smidt-Jensen,' S Lindenberg' \\ 'Copenhagen Fertility Center, Lygten 2c, 2400 Copenhagen, \\ Denmark \\ ${ }^{2}$ Eugin, Carrer de Balmes 236, 08006 Barcelona, Spain
}

Correspondence: Gitte Juul Almind, Copenhagen Fertility Center, Lygten 2c, 2400 Copenhagen, Denmark, Tel +45 3325 7000, Email gittejuul@gmail.com

Received: November 23, 2021 | Published: December 02, 2021

\section{Introduction}

Artificial reproductive technology (ART) aims to achieve best clinical results with the least discomfort and cost. The underlying concept of controlled ovarian hyperstimulation $(\mathrm{COH})$ has been that more oocytes translate into higher success rates as cumulative pregnancy rate (CPR) will increase due to surplus frozen embryos.

The terms natural, patient-friendly, mild, minimal, and minimally stimulating IVF have appeared increasingly in the literature, clinic advertising and the media causing confusion among clinicians, researchers, and patients likewise. The definition of Natural Cycle IVF (NC-IVF) by ISMAAR ${ }^{1}$ is defined as IVF without any medication. The term "modified NC-IVF" includes medication to reduce the likelihood of cycle cancellation, such as human chorionic gonadotropins (hCG) to induce final oocyte maturation and/or gonadotropin releasing hormone $(\mathrm{GnRH})$ antagonists (GnRHant) with or without follicle stimulation hormone (FSH) or human menopausal gonadotropin (hMG). Nargund et al. advocated for minimal stimulation IVF (MSIVF) when generally aimed at retrieving 3-5 oocytes per cycle. ${ }^{1}$ MSIVF comprises a method where FSH is administered at a lower dose and or for a shorter duration in a GnRH-antagonist co-treated cycle.

Minimal stimulation avoids gonadotropin IVF, using clomiphene citrate $(\mathrm{CC})$ tablets $(50 \mathrm{mg} /$ day) from days 3 of menstruation until oocyte trigger (Ovitrelle). ${ }^{2,3}$ Oral anti-estrogenic medicine such as both Tamoxiphene and CC have been re-introduced in ART for several reasons of which the primary reason is safety for the patient. ${ }^{4}$ Tamoxiphene blocks the spontaneous LH surge sufficiently when administrated during the whole length of stimulation, avoiding the need for an antagonist and making everything easier for the patient. ${ }^{5,6}$ Thus, Tamoxiphene provides a simple dual endocrine modulation by stimulating follicular growth and blocking LH surge. Mild and minimal stimulation could be less stressful for the patient and the medication safer and more convenient.

Among clinicians a reluctancy to use these mild approaches ais seen due to doubt as to its clinical effectiveness. This is despite the possible advantages of the mild approach could offer the patient and the society.

With the introduction of elective Single Embryo Transfer (eSET) in IVF as established modus for safety and efficacy in ART the question of how many oocytes is optimal to retrieved might be discussed again. Often forgotten is the fact that the first successful pregnancy achieved with IVF was the result of a natural, unstimulated menstrual cycle. ${ }^{7}$ The combination of mild IVF and eSET Combined with eSET may open the possibility for reducing the patient's discomfort, stress, and cost. ${ }^{8,9}$ The obligation for any physician is to secure and improve health outcomes. Here a milder and more physiological approach to ovarian 
stimulation in IVF cycles might have a place as well as empowering more personal medicine. Japan has embraced minimal stimulation, using protocols developed by Japanese infertility specialist Keiichi Kato in the $1990^{10}$ critics argue that mild stimulation are cheaper than conventional IVF (C-IVF), stating you need more cycles to get the same outcome. ${ }^{2}$

However some data about embryo quality from mild and natural stimulation has indicated a better implantation rate and live birth rate for embryos deriving from mild/minimal/natural stimulation compared to C-IVF. ${ }^{11,12}$

Mild stimulation might be considered in several patients. In 2018 American Society for Reproductive Medicine (ASRM) recommended that poor responders should considerate mild and minimal stimulation. ${ }^{13}$ So far, no study has clearly demonstrated superiority of high-dose protocols over natural or modified NC-IVF in poor responders. Further patient in risk for ovarian hyperstimulation syndrome might also benefit from this.

Mild IVF also offers a cost and availability issue for many patients. Medication costs, clinical services, and laboratory operations contribute to the high cost of conventional IVF. With cost as a major barrier to infertility care, some patients may never have the possibility of getting treatment.

\section{Aim of study}

The aim of this study was to evaluate the efficiency of two different modified cycle natural IVF (NC-IVF) stimulation protocols compared with our conventional IVF (C-IVF) treatment using a short antagonist protocol.

\section{Material and methods}

We performed a retrospective cohort study including all patients admitted to IVF for unexplained infertility, male factor, and tubal factor. All patients were recruited in the same period and allocated to the different treatments on their own request. The study was conducted between June 2019 and February 2021.
We included all women less than 41 years old. Fresh embryo transfer was performed on day two or day three. A pregnancy test (urine hCG) was carried out 14 days after the oocyte retrieval date. Clinical pregnancy was confirmed in the $7^{\text {th }}$ gestational week with presence of gestational sac with yolk sac and foetal echo.

The simple IVF (S-IVF) stimulation protocol: In this protocol, we used only orally administrated Tamoxiphene $40 \mathrm{mg}$. The dose was administrated orally from day three of the cycle until the final oocyte maturation and ovulation. Monitoring ultrasound was initiated on day three and continued until a follicle of $16 \mathrm{~mm}$ was seen. Oocyte retrieval was done 34 hours after the human choriongonadotropin (hCG) trigger (Ovitrelle, Merck)

The mild IVF (M-IVF) stimulation protocol: In this protocol, we used orally administrated Tamoxiphene 40mg and follicle stimulation hormone (FSH). The Tamoxiphene was administrated orally from day three in the cycle and every other day 150 IU FSH were administrated until the final oocyte maturation and ovulation. Monitoring ultrasound was initiated on day three and continued until a follicle of $16 \mathrm{~mm}$ was seen. Oocyte retrieval was done 34 hours after hCG trigger (Ovitrelle, Merck).

The conventional IVF (C-IVF) stimulation protocol: This protocol included 150-200 IU FSH injections daily from day three in the cycle until the follicles had reached a diameter of $17 \mathrm{~mm}$. HCG was given to induce final maturation and ovulation (Ovitrelle, Merck). From the 6th day of FSH stimulation $0.25 \mathrm{mg}$ Orgalutran (SUN Pharma) was administrated daily to avoid premature ovulation. Monitoring ultrasound scans were initiated at day three in the cycle and hereafter until the day of ovulation induction. Oocyte retrieval was done 36 hours after the hCG trigger.

\section{Results}

The study included a total of 976 IVF cycles. 651 cycles from C-IVF, 145 cycles from S-IVF and 180 cycles from M-IVF (Table 1). Mean age in the groups were comparable (C-IVF 35; S-IVF 34 and M-IVF 35 years of age).

Table I Stimulation protocol

\begin{tabular}{|c|c|c|c|c|c|c|c|c|c|}
\hline $\begin{array}{l}\text { Stimulation } \\
\text { protocol }\end{array}$ & $\begin{array}{l}\text { No. } \\
\text { cycle }\end{array}$ & $\begin{array}{l}\text { No. oocyte } \\
\text { retrieval }\end{array}$ & $\begin{array}{l}\text { No. Embryo } \\
\text { Transfer }\end{array}$ & Pregnancy & $\begin{array}{l}\text { Mean } \\
\text { no eggs } \\
\text { retrieved }\end{array}$ & $\begin{array}{l}\text { Mean no } \\
\text { embryos } \\
\text { transferred }\end{array}$ & $\begin{array}{l}\text { Implantation } \\
\text { rate }\end{array}$ & $\begin{array}{l}\text { Pregnancy } \\
\text { rate per } \\
\text { transfer }\end{array}$ & $\begin{array}{l}\text { Pregnancy } \\
\text { Rate per } \\
\text { retrieval }\end{array}$ \\
\hline $\begin{array}{l}\text { Short down } \\
\text { regulation }\end{array}$ & 651 & 651 & 548 & 168 & 6,1 & $\mathrm{I}, \mathrm{I}$ & 30,6 & $29,00 \mathrm{I}$ & 26,00 \\
\hline Simple & 145 & 145 & 87 & 21 & $\mathrm{I}, 2$ & 1 & 22,9 & 25,502 & 14,004 \\
\hline Mild & 180 & 180 & 123 & 25 & 3 & $\mathrm{I}, \mathrm{I}$ & 18,6 & 20,003 & 17,005 \\
\hline
\end{tabular}

T test 2-sided; unpaired:

I Mild versus Simple: $P=0,69$

2 Simple versus Short Downregulation:

$P=0,21$

3 Mild versus Short Downregulation:

$P=0.054$

Chi-square sign. level $p>005$ :

4 Simple versus Short Downregulation:

$P=0,0037$ (Chi-square is 8.39,)

5 Mild versus Short Downregulation:

$\mathrm{P}=0,00 \mathrm{II}(\mathrm{Chi}-$ square is 11,23$)$

Mean number of eggs retrieved was 6.1 (C-IVF), 1.2 (S-IVF) and 3,0 (M-IVF). The total number of embryos transferred was 548 (C-IVF), 87 (S-IVF) and 123 (M-IVF) (Table 1).

The pregnancy rate per transfer was similar in the groups. Clinical pregnancy rate (CPR) per transfer was found to be $29 \%$ for the C-IVF, $25,5 \%$ in the S-IVF group and for the M-IVF $18,6 \%$ (Table 1 ). The

implantation rate 30,6\% (C-IVF), 22,9\% (S-IVF) and 18,6\% (M-IVF) (Table 1).

FSH units use (Figure 1) was found to be considerably less in the mild stimulation compared to conventional IVF. C-IVF 1650IU FSH; M-IVF 650IU FSH. 


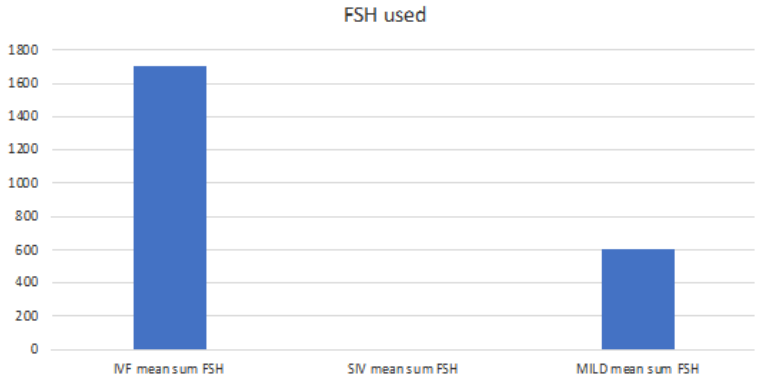

Figure I Units of FSH used per stimulation cycle.

\section{Discussion}

The data from the present retrospective cohort study demonstrates that the clinical outcome from two types of modified NC-IVF cycles was comparable with the C-IVF protocol when pregnancy rate per fresh transfer was the primary outcome.

Despite significant advancements in the field, the process of human reproduction remains inefficient with many unanswered questions. C-IVF aims to retrieve many oocytes to maximize the number of embryos available for ET or cryopreservation (cumulative pregnancy rates). Van der Gaast reported the optimal number of oocytes associated with the optimal chance of conceiving after ET to be $13 .{ }^{14}$ The paradox remains that despite the practice of producing multiple embryos most embryos do not implant or do not result in a live birth.

In Scandinavia, eSET has been practiced for many years. This makes it unnecessary to harvest more than a few eggs in one IVF cycle when fresh transfer is used predominantly. SET policy reduces embryo wastage in regular IVF and can therefore be an attractive concept for countries with strict legislation that forbids the destruction of surplus embryos, both fresh and frozen.

Patients going for these modified NC-IVF protocols need to be informed and counselled properly about the aim to harvest very few follicles and therefore the risk of repeated cycles.

The M-IVF and S-IVF protocols could be an option for all patients but women at risk of ovarian hyperstimulation syndrome (OHSS). Furthermore randomized studies have demonstrated a trend towards a higher proportion of good-quality embryos/blastocysts when using low FSH stimulation protocols. ${ }^{15,16}$

Another emerging concern is the impact of stimulation and longtime culture of embryos to blastocyst on the epigenetic changes during ART. ${ }^{17}$ Therefore, the concern for ART offspring might enable a place for milder stimulation in the future.

Two randomised clinical trials (RCTs) compared natural IVF with conventional IVF in poor responders. Morgan found no difference in cumulative pregnancy rate (CPR) and a better implantation rate (IR) in NC-IVF. Another from Kim et al. RCT $(n=90)$ compared modified NC-IVF with high FSH dose antagonist protocol and found no difference in the live birth rate (LBR). ${ }^{18,19}$

Two meta-analyses reported lower ongoing pregnancy rates with very mild stimulation. Matsaseng compared different mild stimulations. Four studies using $150 \mathrm{IU}$ FSH and GnRH antagonist and the fifth using clomiphene citrate (CC) and 75 IU FSH and compared it to C-IVF. ${ }^{20,21}$

Verberg et al. analysed three RCTs comparing the efficacy of the mild ovarian stimulation regimen (involving midfollicular phase initiation of FSH and GnRH co-treatment) for IVF with a conventional long GnRH agonist co-treatment stimulation.
In a recent review, $20 \mathrm{RCT}$ were identified and most claimed mild stimulation to be equal to conventional IVF in pregnancy rate per ET. ${ }^{22}$ Results from a large study with more than 20,000 cycles from minimal stimulation and natural cycle IVF show a similar level of pregnancy rates when compared to C-IVF treatment. ${ }^{2}$

Among normal responders in IVF, the use of low-dose FSH (150IU/ day) with or without oral agent (CC or letrozole) results in clinical pregnancy rates (CPR's) and live birth rates (LBRs) comparable to those with C-IVF. ${ }^{23}$

In our clinic, we use the NC-IVF stimulations for women at risk of OHSS or when the women prefer less hormone or lower price for IVF. The evidence on cycle cancellation is conflicting and some metaanalyses of RCTs showed higher cumulative pregnancy rate with mild IVF $^{24-27}$ while other meta-analyses found no difference. ${ }^{23,28}$

One study outlines that affordable ART with low dose stimulation provide a more patient-friendly treatment for many women undergoing fresh IVF treatment cycles because they cause less stress and are more convenient for the patient. ${ }^{22}$ Overall, this study suggested that modified NC-IVF is a good alternative.

World Health Organisation (WHO) has recognised infertility as a global health issue and outlined proposal to deliver a standard and affordable fertility care. Therefor mild IVF might be further developed an may in the future find a place to make IVF more universally available and affordable. ${ }^{29-31}$ ESET combined with simple or mild stimulation may be an important step towards these goals. Further reduction in $\mathrm{n}$ in multiple gestation rates should be mentioned.

However, limitations of the present study are related to the relatively small number of cycles and the retrospective nature of the study. Large prospective studies need to be performed to give the final answer whether these NC-IVF protocols will become the first line option in IVF programmes.

\section{Conclusion}

In conclusion, the clinical pregnancy rates per eSET were comparable in our centre. The results of number of cycles are still low.

Furthermore, these modified NC-IVF protocols seem to be a "low risk" and "low-cost" option in an IVF programme. They could become the first choice of treatment for many patients in the future and a good alternative to IUI. In addition, the two main complications associated with the use of ART as OHSS and multiple pregnancies, could be eliminated by NC-IVF stimulation protocols and the increased use of an eSET policy.

\section{Acknowledgments}

None.

\section{Author contribution statement conception and design}

All authors; Collection and assembly of data: GJ. Almind, S. Lindenberg; Data analysis and interpretation: all authors. Manuscript writing: All authors; (VII) Final approval of manuscript: All authors. Keywords: ART, IVF, Mild IVF, Natural IVF, cost, stress, results, retrospective, single embryo transfer.

\section{Ethics statements studies involving animal subjects generated statement}

No animal studies are presented in this manuscript. Studies involving human subjects Generated Statement: This study us a 
retrospective study on anonymized data. Inclusion of identifiable human data is not identified. Generated Statement: No potentially identifiable human images or data is presented in this study.

\section{Funding}

Self-funded.

\section{Conflicts of interest}

None of the authors has reported any conflicts of interest. Any external funding did not support this study.

\section{References}

1. Nargund G, Fauser B C J M, Macklon N S, et al. The ISMAAR proposal on terminology for ovarian stimulation for IVF. Hum Reprod. 2007;22:2801-2804.

2. Kato K, Takehara Y, Segawa T, et al. Minimal ovarian stimulation combined with elective single embryo transfer policy: Age-specific results of a large, single-centre, Japanese cohort. Reprod Biol Endocrinol. 2012;10:1-7

3. Teramoto S, Kato O. Minimal ovarian stimulation with clomiphene citrate: a large-scale retrospective study. Reprod Biomed Online. 2007;15:134 148.

4. Branigan EF, Estes MA. Minimal stimulation IVF using clomiphene citrate and oral contraceptive pill pretreatment for LH suppression. Fertil Steril. 2000;73:587-590.

5. B Lindenberg F. Low ovarian stimulation using tamoxifen/FSH compared to conventional IVF: a cohort comparative study in conventional IVF treatments. Reprod Syst Sex Disord. 2012.

6. Almind G, Faerch E, Lindenberg F, et al. Mild stimulation approach for in vitro fertilization treatment: retrospective data from one Danish Centre Facts, views Vis. ObGyn. 2018;10:81-84.

7. Steptoe PC, Edwards RG. Birth after the reimplantation of a human embryo. Lancet. 198;312:366.

8. Højgaard A, Ingerslev HJ, Dinesen J. Friendly IVF: patient opinions. Hum Reprod. 2001;16:1391-1396.

9. de Klerk C, Macklon NS, Heijnen E M E W, et al. The psychological impact of IVF failure after two or more cycles of IVF with a mild versus standard treatment strategy. Hum Reprod. 2007;22:2554-2558.

10. Abe T, Yabuuchi A, Ezoe K, et al. Success rates in minimal stimulation cycle IVF with clomiphene citrate only. J Assist Reprod Genet. 2020;37:297-304.

11. Farquhar C, Marjoribanks J, Brown J, et al. Management of ovarian stimulation for IVF: narrative review of evidence provided for World Health Organization guidance. Reproductive BioMedicine Online. 2017;35:3-16.

12. Martin JR, Bromer JG, Sakkas D, et al. Live babies born per oocyte retrieved in a subpopulation of oocyte donors with repetitive reproductive success. Fertil Steril. 2018;94:2064-2068.

13. Committee P, Society A. Comparison of pregnancy rates for poo responders using IVF with mild ovarian stimulation versus conventional IVF: a guideline. Fertil Steril. 2018;109:993-999.

14. van der Gaast MH, Eijkemans MJC, van der Net JB, et al. Optimum number of oocytes for a successful first IVF treatment cycle. Reprod Biomed Online. 2006;13:476-480.
15. Casano S, Guidetti D, Patriarca A, et al. MILD ovarian stimulation with GnRH-antagonist vs. long protocol with low dose FSH for non-PCO high responders undergoing IVF: a prospective, randomized study including thawing cycles. J Assist Reprod Genet. 2012;29:1343-1351.

16. Hohmann FP, Macklon NS, Fauser BCJM. A randomized comparison of two ovarian stimulation protocols with gonadotropin-releasing hormone $(\mathrm{GnRH})$ antagonist cotreatment for in vitro fertilization commencing recombinant follicle-stimulating hormone on cycle day 2 or 5 with the standard long GnRH a. J Clin Endocrinol Metab. 2003;88:166-173.

17. Pinborg A, Loft A, Romundstad LB, et al. Epigenetics and assisted reproductive technologies. Acta Obstet Gynecol Scand. 2016;95:10-15.

18. Morgia F, Sbracia M, Schimberni M, et al. A controlled trial of natural cycle versus microdose gonadotropin-releasing hormone analog flare cycles in poor responders undergoing in vitro fertilization. Fertil Steril. 2004;81:1542-1547.

19. Kim $\mathrm{CH}$, Kim SR, Cheon YP, et al. Minimal stimulation using gonadotropin-releasing hormone $(\mathrm{GnRH})$ antagonist and recombinant human follicle-stimulating hormone versus GnRH antagonist multipledose protocol in low responders undergoing in vitro fertilization intracytoplasmic sperm injectio. Fertil Steril. 2009;92:2082-2084.

20. Matsaseng T, Kruger T, Steyn W. Mild ovarian stimulation for in vitro fertilization: are we ready to change? A meta-analysis. Gynecol Obstet Invest. 2013;76:233-240.

21. Verberg MFG, Macklon NS, Nargund G, et al. Mild ovarian stimulation for IVF. Hum Reprod Update. 2009;15:13-29.

22. Nargund G, Datta AK, Fauser BCJM. Mild stimulation for in vitro fertilisation. Fertil Steril. 2018;108:558-567.

23. Datta AK, Maheshwari A, Felix N, et al. Mild versus conventional ovarian stimulation for IVF in poor, normal and hyper-responders: a systematic review and meta-analysis. Hum Reprod Update. 2021;27:229-253.

24. Kamath MS, Maheshwari A, Bhattacharya S, et al. Oral medications including clomiphene citrate or aromatase inhibitors with gonadotropins for controlled ovarian stimulation in women undergoing in vitro fertilisation. Cochrane Database Syst Rev. 2017.

25. Wago KJ, Skarsvåg TI, Lundbom JS, et al. The importance of needle gauge for pain during injection of lidocaine. J Plast Surg Hand Surg. 2016;50:115-118

26. Youssef MAF, van Wely M, Mochtar M, et al. Low dosing of gonadotropins in vitro fertilization cycles for women with poor ovarian reserve: systematic review and meta-analysis. Fertil Steril. 2018;109:289-301.

27. Montoya-Botero P, Drakopoulos P, González-Foruria I, et al. Fresh and cumulative live birth rates in mild versus conventional stimulation for IVF cycles in poor ovarian responders: a systematic review and meta-analysis. Hum Reprod Open. 2021:1-10.

28. Fan Y, Zhang X, Hao Z, et al. Effectiveness of mild ovarian stimulation versus $\mathrm{GnRH}$ agonist protocol in women undergoing assisted reproductive technology: a meta-analysis. Gynecol Endocrinol. 2017;33:746-756.

29. Nargund G, Fauser BCJM. Mild ovarian stimulation for IVF is the smartest way forward. Reproductive biomedicine online. 2020;41:569-571.

30. Ombelet W. WHO fact sheet on infertility gives hope to millions of infertile couples worldwide. Facts views Vis ObGyn. 2020;12:249.

31. Chambers GM, Fauser BCJM. Access to ART treatment and gender equality. Reproductive Biomed Online. 2021;42:687-690. 